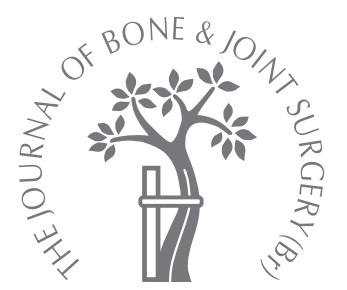

L. C. Hamilton, L. C. Biant, L. N. Temple, R. E. Field

From the South West

London Elective

Orthopaedic Centre, Epsom, England

L. C. Hamilton, MA Oxon, MRCS, Senior House Officer - L. C. Biant, FRCSEd(Tr \& Orth), MS, Hip and Knee Fellow - R. E. Field, PhD, FRCS, FRCS(Orth), Consultant Orthopaedic Surgeon and Director of Research South West London Elective Orthopaedic Centre

L. N. Temple, FRCPath, Consultant Histopathologist Epsom and St Helier Hospitals Dorking Road, Epsom, Surrey KT18 7EG, UK.

Correspondence should be sent to Ms L. C. Biant; e-mail: leela. Biant@luht.scot.nhs.uk

(C)2009 British Editorial Society of Bone and Joint Surgery doi:10.1302/0301-620X.91B4. $21707 \$ 2.00$

$J$ Bone Joint Surg $[\mathrm{Br}]$ 2008;91-B:533-5. Received 28 August 2008; Accepted after revision 17 December 2008

\title{
Isolated pseudogout diagnosed on hip arthroscopy
}

\author{
Idiopathic calcium pyrophosphate deposition disease (pseudogout) has a variable \\ presentation. Many joints are usually affected; single joint disease is uncommon. We \\ present a case report of primary monoarticular pseudogout affecting the hip. The diagnosis \\ was made on the appearance and analysis of specimens obtained at arthroscopy. \\ Monoarticular pseudogout is rare, but should be considered in the differential diagnosis of \\ any presentation of joint pain.
}

Calcium pyrophosphate deposition disease usually manifests as a polyarticular arthritis, although it can initially present as a monoarticular disorder affecting the knees, shoulders, wrists and fingers. However, cases have been reported affecting such varied sites as the cervical spine, elbow, pubic symphysis and temporomandibular joint. ${ }^{1-3}$ Presentation may resemble that of rheumatoid arthritis, ${ }^{4}$ septic arthritis, osteoarthritis or a Charcot joint. ${ }^{5}$ Chondrocalcinosis of the hip is usually only seen as part of a widespread polyarthropathy, which can be associated with rapid degeneration of the joint. ${ }^{6}$ We present a case of primary monoarticular pseudogout affecting the hip.

\section{Case Report}

A healthy 56-year-old fireman and professional squash coach was referred with a threeyear history of intermittent left groin pain of progressively increasing severity, exacerbated by exercise. There was no history of trauma. His sporting activities had become restricted by pain, and he had been obliged to retire as a professional sportsman. Two years previously, local anaesthetic and steroid had been injected into the left greater trochanteric area, without symptomatic improvement. One year later, local anaesthetic and steroid had been injected into the hip under radiological guidance as a diagnostic procedure, but once more his symptoms persisted.

Examination of the hip showed a fixed flexion deformity of $10^{\circ}$ with extension apparently restricted by pain. The range of movement was otherwise full and symmetrical with that of the right hip. Pain was elicited on flexion, internal and external rotation of the left hip. Plain radiographs (Fig. 1), a hip arthrogram, CT scan and MR arthrogram were all normal. There was no clinical evidence of a hernia or nerve root entrapment.

At arthroscopy (Fig. 2) there was good visualisation of both central and peripheral compartments of the hip. The articular cartilage of the femoral head was in pristine condition. In contrast, the labrum and articular cartilage of the acetabulum had a diffuse, white speckled appearance consistent with crystal deposition or previous steroid injection. The labrum was intact, with no evidence of femoroacetabular impingement on moving the hip. There were no loose bodies. Biopsies of the labrum and acetabular cartilage were sent for histological examination. Attempts were made to remove the deposits using the arthroscopic shaver and a probe, however as this might have damaged the articular surface and labrum, the joint was thoroughly lavaged instead.

Histology of the acetabular cartilage showed fibroconnective tissue containing crystalline deposits which demonstrated weakly positive birefringence in keeping with pseudogout (Fig. 3). Serum liver function, bone profile (without uric acid level test), haematological and thyroid function tests were normal, thus excluding other conditions which can lead to crystal deposition.

Arthroscopy provided a diagnosis, but joint lavage and partial debridement failed to improve the patient's symptoms. He continues to suffer from episodes of pain, which have been managed predominantly with antiinflammatory medication. 


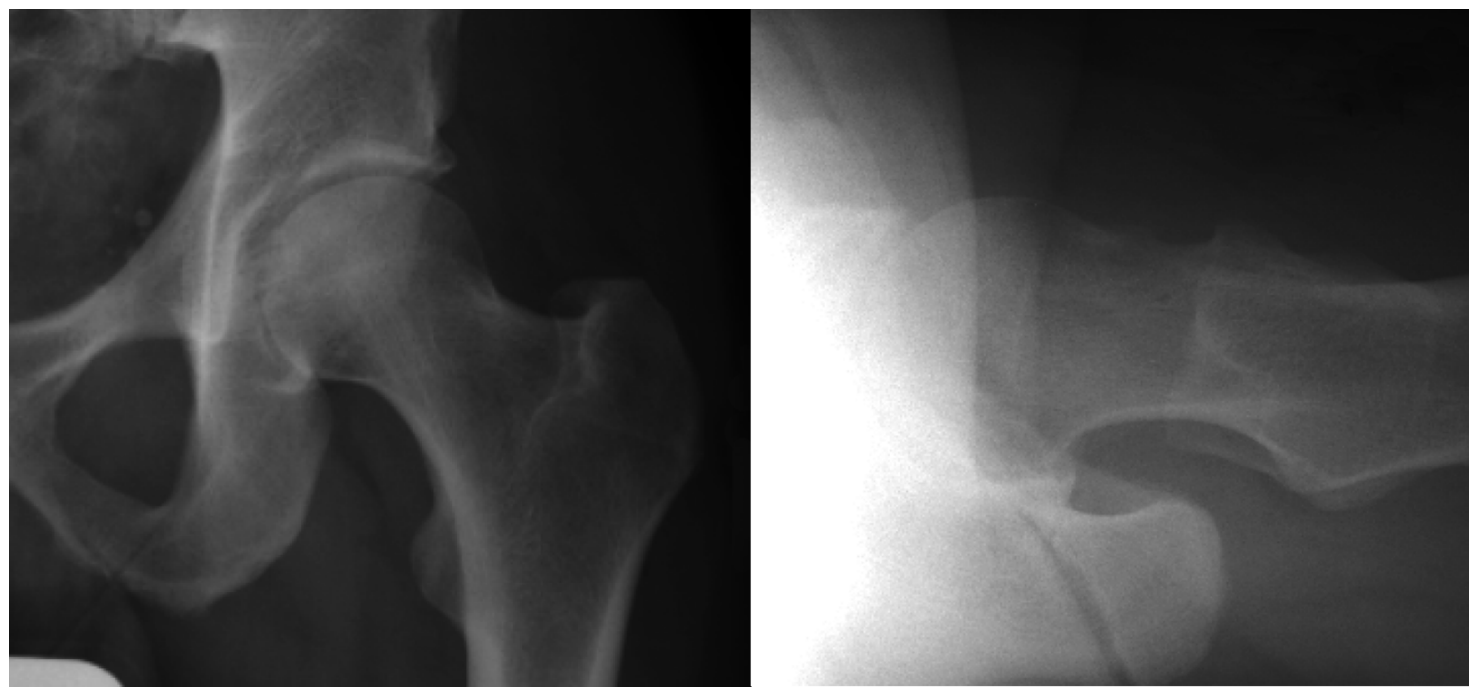

Fig. 1

Anteroposterior and lateral plain radiographs of the left hip.

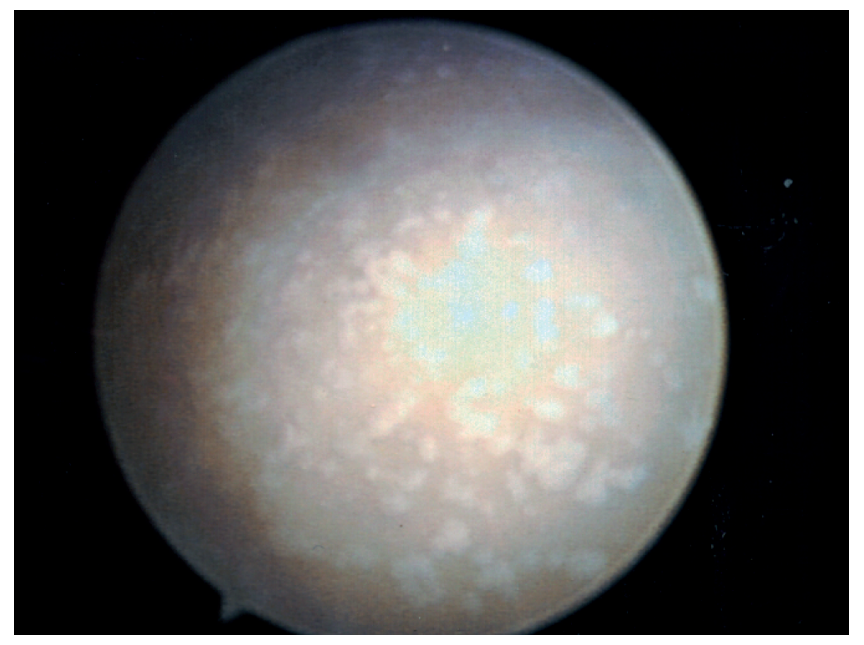

Fig. 2a

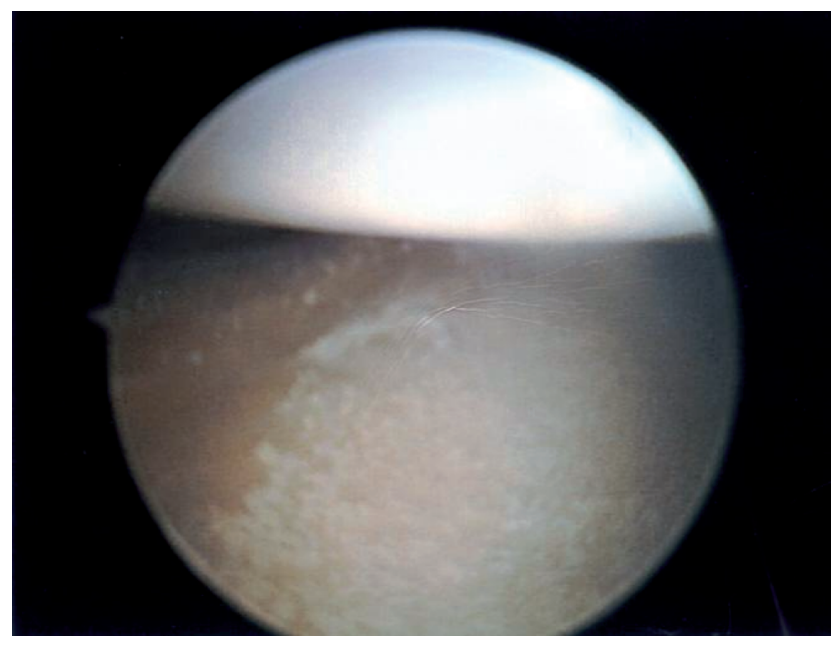

Fig. $2 b$

Arthroscopic view of the hip showing a) the white speckled appearance of the acetabular articular surface and b) the femoral articular surface shown superiorly with an entirely normal macroscopic appearance.

\section{Discussion}

'Pseudogout' refers to inflammation of a joint that may resemble gout, but is not caused by negatively birefringent monosodium urate crystals. 'Chondrocalcinosis' describes calcification within fibrous or hyaline cartilage structures, diagnosed on imaging as a dense line parallel to the articular surface, ${ }^{7}$ and can be a feature of pseudogout. Calcium pyrophosphate deposition disease is the histological diagnosis, with clinical manifestations resulting from precipitation of calcium pyrophosphate dihydrate crystals in the articular tissue. These crystals are rod- or rhomboid-shaped and characterised by weak or absent positive birefringence on polarising microscopy.
The detailed pathophysiology of pseudogout has not been fully elucidated. ${ }^{8-11}$ Calcium pyrophosphate crystal deposition is more common in older women with osteoarthritis. It can also be related to haemochromatosis, hypomagnesaemia, hyperparathyroidism, hypophosphataemia, Wilson's disease and joint damage due to hypothyroidism. However, there have been isolated cases of young patients developing calcium pyrophosphate deposition disease with no associated diseases or familial occurrence. ${ }^{2,12}$

In this case, a relatively young, healthy man with no arthritis or metabolic disorder developed isolated pseudogout of the hip. It remains unclear why the crystals were only present on the acetabular articular surface. We believe 


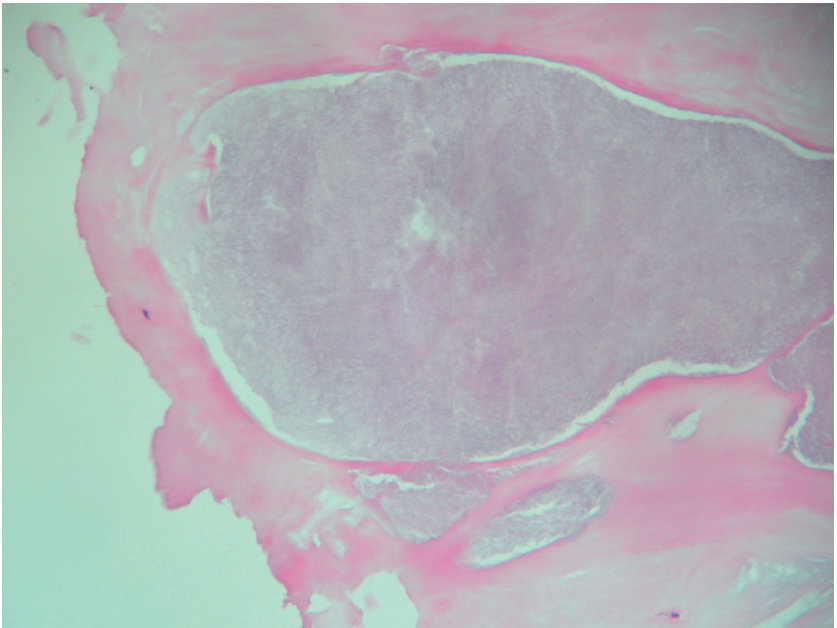

Fig. 3a

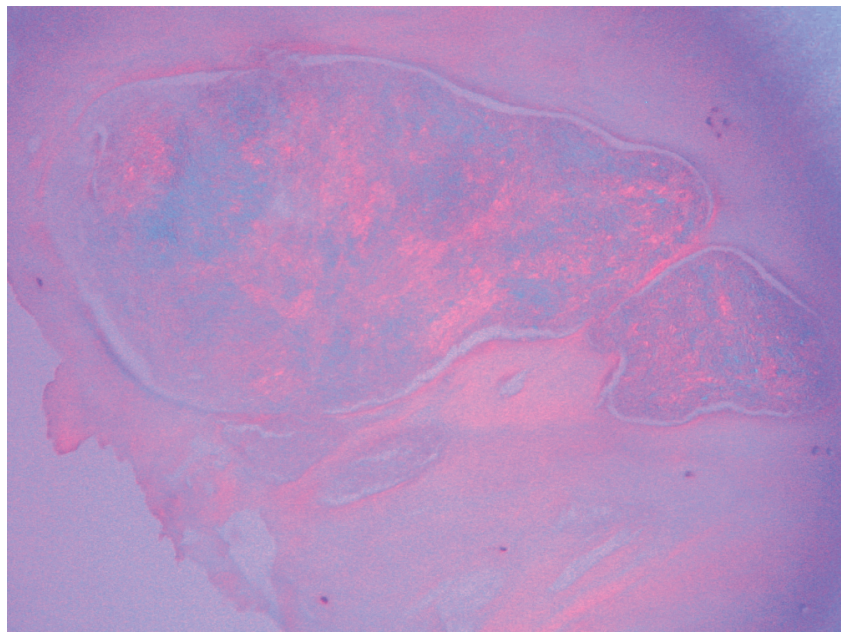

Fig. 3b

(a and b) Histology of the acetabular cartilage with haematoxylin and eosin staining showing amorphous granularity typical of crystal deposition (magnification $\times 250$ ).

this is the first reported case of primary monoarticular pseudogout in the hip. The intermittent nature of the pain and the inability of the imaging and diagnostic injections to identify an abnormality is not unusual in pseudogout. ${ }^{8}$ Hip arthroscopy has been shown to be most useful in diagnosing chondral lesions ${ }^{13}$ and it proved invaluable in the diagnosis of this unusual cause of hip pain. However, as this case suggests, lavage and debridement of the joint may not necessarily give relief of symptoms. There is little convincing evidence in the literature of the therapeutic benefit of lavage in other joints with pseudogout. One study ${ }^{14}$ reported good results when lavage was used for crystal disease in the knee, but the patients had a large array of other pathology that could have caused symptoms. Antiinflammatory medication remains the mainstay of treatment for symptomatic pseudogout.

No benefits in any form have been received or will be received from a commercial party related directly or indirectly to the subject of this article.

\section{References}

1. Fye KH, Weinstein PR, Donald F. Compressive cervical myelopathy due to calcium pyrophosphate dehydrate deposition disease: report of a case and review of the literature. Arch Intern Med 1999;159:189-93.

2. Hayashi M, Matsunaga T, Tanikawa H. Idiopathic widespread calcium pyrophosphate dihydrate crystal deposition disease in a young patient. Skeletal Radiol 2002;31:246-50.
3. Ishida T, Dorfman HD, Bullough PG. Tophaceous pseudogout (tumoral calcium pyrophosphate dehydrate crystal deposition disease). Hum Pathol 1995;26:587-93.

4. Song JS, Lee YH, Kim SS, Park W. A case of calcium pyrophosphate dihydrate crystal deposition disease presenting as an acute polyarthritis. J Korean Med Sci 2002;17:423-5.

5. Menkes CJ, Chouraki L. Clinical manifestations of joint chondrocalcinosis. Rev Prat 1994;44:183-8 (in French).

6. Menkes CJ, Decraemere W, Postel M, Forest $\mathbf{M}$. Chondrocalcinosis and rapid destruction of the hip. J Rheumato/ 1985;12:130-3.

7. Bencardino JT, Hassankhani A. Calcium pyrophosphate dihydrate crystal deposition disease. Semin Musculoskelet Radiol 2003;7:175-85.

8. Wright GD, Doherty M. Calcium pyrophosphate crystal deposition is not always 'wear and tear' or aging. Ann Rheum Dis 1997;56:586-8.

9. Ishikawa K, Higashi I, Shimomura Y, Yonemura K. A histological study of calcium pyrophosphate dihydrate crystal-deposition disease. J Bone Joint Surg [Am] 1989;71-A:875-86

10. Dijkgraaf LC, Liem RS, de Bont LG, Boering G. Calcium pyrophosphate dihydrate crystal deposition disease: a review of the literature and a light and electon microscopic study of a case of the temporomandibular joint with numerous intracellular crystals in the chondrocytes. Osteoarthritis Cartilage 1995;3:33-45.

11. Reginato AM, Olsen BR. Genetics and experimental models of crystal-induced arthritis: lessons learned from mice and men: is it crystal clear? Curr Opin Rheumatol 2007; 19:134-45

12. Hammoudeh M, Siam AR. Pseudogout in a young patient. Clin Rheumatol 1998;17:242-5.

13. Baber YF, Robinson AH, Villar RN. Is diagnostic arthroscopy of the hip worthwhile?: a prospective review of 328 adults investigated for hip pain. J Bone Joint Surg [Br] 1999;81-B:600-3

14. O'Connor RL. The arthroscope in the management of crystal induced synovitis of the knee. J Bone Joint Surg [Am] 1973;55-A:1443-9. 